\title{
Side Branch Interaction with Main Line Standing Waves and Related Signal Handling Approaches
}

\author{
Arthur Ruggles, Eric M. Moore, and Michael Shehane \\ Department of Nuclear Engineering, University of Tennessee, Knoxville, TN 37996-2300, USA \\ Correspondence should be addressed to Arthur Ruggles; aruggles@utk.edu
}

Received 27 July 2012; Revised 18 October 2012; Accepted 25 February 2013

Academic Editor: Tamer Elnady

Copyright ( 2013 Arthur Ruggles et al. This is an open access article distributed under the Creative Commons Attribution License, which permits unrestricted use, distribution, and reproduction in any medium, provided the original work is properly cited.

\begin{abstract}
Data from a low pressure air test facility are used to quantify the influence of the acoustic field in the main line on side branch resonance behavior. The main line of diameter $D=7.6 \mathrm{~cm}$ may accumulate acoustic energy broadcast from a resonating branch of diameter $d=1.9 \mathrm{~cm}(d / D=0.25)$. The side branch resonance amplitude is a strong function of branch position along the main line with the normalized pressure rising to 1.2 in the most favorable branch positions with Strouhal number near 0.3. Large time variation of the side branch and main line resonance amplitude is apparent for most branch positions. A moving window is used on the time history to collect an array of power spectral densities (PSDs). Peak amplitude values from the PSD array are represented in a probability density function (PDF) that provides a repeatable characterization of data from the system.
\end{abstract}

\section{Background}

Small branch lines off larger main delivery lines can exhibit acoustic resonance. Branch lines with a reflective obstruction, such as a valve or instrument, are susceptible to a $1 / 4$ wavelength standing wave, with a pressure node positioned near where the branch meets the main line, and a pressure antinode positioned at the obstruction. Of course, higher modes are possible with an odd number of quarter wavelengths existing in the branch such that

$$
f_{\text {natural }}=\frac{n c}{4 L} \quad n=1,3,5,7, \ldots,
$$

where $c$ is the sound speed and the characteristic length, $L$, is the branch length.

Vorticity in the fluid near the wall of the main line becomes free at the branch and diverts flow periodically into the downstream branch. This in concert with a side branch resonance near the vortex frequency sustains a standing wave in the branch, Rockwell and Naudascher [1]. The frequency for the vortex formation is predicted using the Strouhal number, St $=f d / v$, with the branch diameter providing the length scale and $v$ set to the flow velocity in the main line. Coupling between the branch acoustic response and the vortex shedding behavior allows for a branch to resonate for values of Strouhal number ranging roughly from 0.2 to 0.6 , Ziada and Shine [2]. Strouhal number near 0.4 normally gives the largest amplitude acoustic response in the branch.

The position of the branch downstream of a fitting such as a valve, orifice, or elbow has been shown to influence vortex shedding and the associated Strouhal number associated with peak response, Ziada and Shine [2] and Lamoureux and Weaver [3]. More than one vortex can span the branch opening, leading to excitation of fundamental acoustic frequencies in branch lines at higher Strouhal numbers, Ziada [4]. Branches positioned across from each other or in tandem may interact to enhance the acoustic response of the system, Ziada and Buhlmann [5]. Modifications to the geometry of the junction of the branch to the main line have been explored to mitigate vortex shedding as a coherent source of energy for driving branch line resonance, Weaver and McLeod [6].

Acoustic coupling of branch line modes through the main line has been studied by Coffman and Bernstein [7], Ziada and Buhlmann [5], Ziada and Shine [2], and Kriesels et al. [8]. Acoustic power radiation from the branch into the main line is normally the main sink for the energy driving the branch resonance, Bruggeman [9] and Kriesels et al. [8]. Acoustic amplitudes in the branch are enhanced when coupling between branches reduces the net power radiated into the main line from the branches. 
In circumstances where damping is low, and the acoustic energy in the main line has limited opportunity for escape, the acoustic power propagated into the main line from the branch can accumulate in the main line. Standing waves in main lines are known to influence vortex shedding meter performance, Peters et al. [10]. Standing waves in main flow lines may load flow components and have fatigued steam dryers in nuclear power plant steam supplies [11]. Pressure oscillations created in the main line near the branch resonance frequency and branch location can influence the classical branch resonant frequency from (1). The branch acoustic amplitude is also altered by the acoustic energy in the main line and can be driven by the main line acoustic energy according to acoustic circuit theory, even in the absence of vortex shedding. Most literature treats branch resonance for the case where the main line is engineered to be an acoustic sink. Coupling of the main line acoustic waves with the branch resonance causes branch acoustic behavior not observed when the main line does not accumulate acoustic energy. This paper presents experiments and data for branch resonance coupled with main line standing waves. A method to condense and quantify the nonstationary acoustic data in a repeatable manner is also provided. Long time histories of up to two minutes duration are broken into shorter time segments, and the shorter time segments are transformed into frequency domain using power spectral densities (PSDs). The resonance peaks from the array of PSD from the moving time windows are then represented in a probability density function (PDF) to represent the non-stationary attributes of the longer time history. This statistical representation of the resonance peaks from the frequency domain is convenient for use as a load representation for structural mode response assessment and stress prediction.

\section{Experiment and Data Characterization}

An atmospheric pressure air test facility was developed to examine basic attributes of the relationship between standing waves in the main line and the branch line. The experiment was motivated by features observed in data collected on main steam lines in nuclear power plants that suggested the acoustic waves in the main steam lines play a role in the branch resonance behavior. The experiment is designed to maintain the ratio of branch diameter, $d$, over main line diameter, $D$, equal to 0.25 , consistent with that of the nuclear steam supply, with the air experiment at near one over eight geometric scale. The flow Mach number is near 0.1, which is also close to that in the nuclear steam supply. A branch of diameter $d=1.9 \mathrm{~cm}$ with length $16 \mathrm{~cm}$ is mounted on a flow line of diameter $D=7.6 \mathrm{~cm}$, and velocities are tested providing Strouhal numbers from 0.2 to 0.3 . The flow dynamic pressure ranges near $900 \mathrm{~Pa}$ for most tests. The peak normalized pressure, defined as the acoustic pressure over flow dynamic pressure, rises to 1.2 for the most favorable branch locations for resonance.

2.1. Experimental Facility: General. The experimental facility consists of a main line $297 \mathrm{~cm}$ long made of sections of $7.6 \mathrm{~cm}$ (3 in) inner diameter schedule 40 PVC piping. The main line

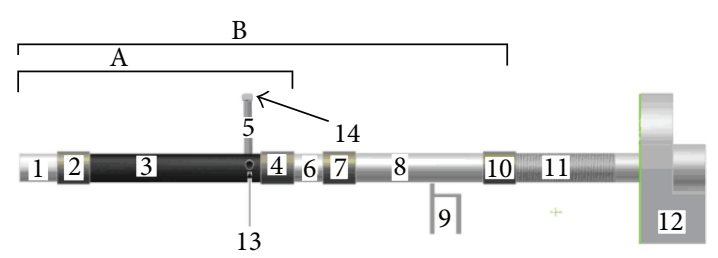

FIGURE 1: Waveguide schematic.

exit is connected to the suction of a Dayton model 3N669A $3.7 \mathrm{~kW}$ blower via flexible corrugated thin wall aluminum tubing. A schematic of the facility is shown in Figure 1, with photographs following in Figure 2. The facility has assumed many configurations during the testing campaign, and Figure 2(a) shows the facility in the normal location under a table supporting data acquisition components, with bubble wrap used for vibration isolation. Figure 2(a) includes a long branch of variable active length used in early testing. Figure 2(b) shows the waveguide configuration used for data reported here, but with the waveguide moved from under the table and supported temporarily on pipe stubs for photographs.

Air was drawn through the apparatus from section 1 to 12 . The air inlet is cantilevered into the room, and no converging inlet section is employed. Main line flow Reynolds numbers are near 2 million for these tests, and flow separation and turbulence are expected in the inlet. This provides acoustic and flow inlet conditions consistent with many fluid transport situations. Prior studies have examined side branch performance downstream of elbows and fittings [2, $3]$, and shift in the Strouhal number at peak excitation and in resonance strength was attributed to perturbation of bulk flow distribution and the velocity profile near the wall. Those effects grow weak for branch positions approaching 10 main line diameters downstream. Side branch locations were investigated starting from $55 \mathrm{~cm}$ (i.e., 7 main line diameters) downstream of the inlet for these tests which is downstream of the region where inlet flow is separated. Broad band flow noise was observed in frequencies less than $100 \mathrm{~Hz}$.

The blower has constant speed, and an air bypass valve used to reduce facility flow is visible to the right of Figure 2. Velocities up to $43 \mathrm{~m} / \mathrm{s}$ are possible. Sections $1,3,6$, and 8 are interchangeable so that the position of the branch line can be changed with respect to the upstream and downstream acoustic boundaries. The total length of the main line can also be varied. The upstream boundary, positioned left of section 1, was open to the room and contained no obstructions for several diameters in every direction. The downstream transition from PVC piping (section 10) to thin wall flexible corrugated aluminum tubing (section 11) was acoustically compliant. Sections 2, 4, 7, and 10 show schedule 40 PVC couplings. Section 5 represents a short capped branch line made of $1.9 \mathrm{~cm}$ (3/4 in) schedule $40 \mathrm{PVC}$ pipe. The branch is prepared by drilling a hole through the pipe and solvent welding the branch in the hole to create a sharp edged branch as shown in Figure 3. The apparatus contains 2 through wall mounted PCB Piezotronics microphones, located at positions 13 and 14 in Figure 1, and another microphone on measuring 


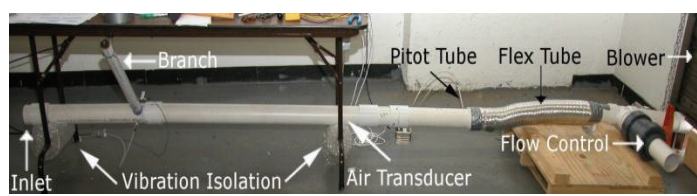

(a)

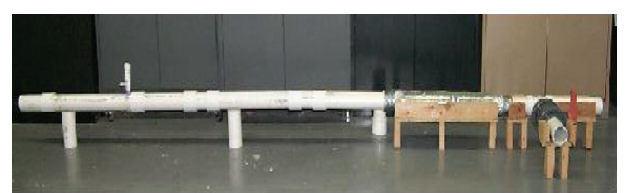

(b)

FIGURE 2: (a) Early waveguide configuration positioned under table for operation. Waveguide is supported on bubble wrap vibration isolators, and early long variable active length branch is depicted. (b) photograph of main line, branch and bypass valve, without blower. Waveguide is supported on pipe stubs to facilitate photograph.

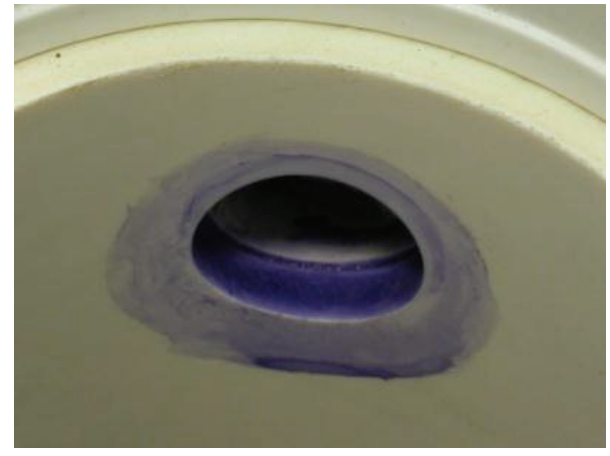

FIGURE 3: A View from the inside of the pipe of a sharp edge branch.

tape that could be moved in the main line. Sometimes the microphone in the branch was positioned $5 \mathrm{~cm}$ up from the junction with the main line to reduce sound pressure levels and keep the microphone in range. That microphone position can be seen in Figure 2(b). Several model microphones were employed depending on required sensitivity, including models 130B10 and 130D20, running through a four-channel ICP signal conditioner. Model 377B10 and model 377A12 microphones were connected to model $426 \mathrm{~B} 03$ preamps. A pitot tube was used to measure fluid velocity and is shown at position 9. It was connected to a Dwyer model MS331 Magnesense differential pressure transmitter that had a response time of $0.5 \mathrm{~s}$. An Omega FMA-905-V film sensor was also used for velocity measurement for low velocity testing, and a Dwyer 641-6-LED film probe was used for the higher velocity testing. Dynamic pressure sample rates up to $100 \mathrm{kS} / \mathrm{s}$ were possible using a National Instruments DAQ model NI USB-9162. Sample rates of 5,000 samples per channel were most often used. Flow velocity data were collected at the same sample rate as the dynamic pressure, and inlet air temperature is measured and recorded with each data set.

The side branch acoustic energy propagates into the main line flow, leading to upstream and downstream waves of common frequency relative to the laboratory reference. However, waves traveling upstream from the branch in the main line are of shorter wavelength than those traveling downstream from the branch in the main line due to the main line flow. The main line has a reflective boundary at the entrance and where the rigid pipe meets the corrugated aluminum exit line; so, waves injected at the branch location are reflected at the main line ends. The acoustic behavior in the main line was explored for varying main line lengths in previous work [12]. The main line and branch acoustic amplitudes are not stationary for the majority of the system configurations that were tested.

The schedule 40 PVC pipe compliance reduces the air sound speed, $C_{\text {gas }}$, in the main line. The effective main line one dimensional sound speed, $C_{\mathrm{ml}}$, may be approximated as

$$
C_{\mathrm{ml}}=C_{\mathrm{gas}} \sqrt{\frac{1}{1+D \rho C_{\mathrm{gas}}^{2} /[\delta E]}},
$$

where $D$ is the pipe diameter, $\delta$ is the pipe wall thickness, $E$ is the pipe material modulus, and $\rho$ is the air density. The sound speed for air at STP is taken as $343.2 \mathrm{~m} / \mathrm{s}$, the PVC schedule 40 wall thickness is $5.8 \mathrm{~mm}$ (0.229 in.), and the PVC modulus is taken as $3,000 \mathrm{MPa}$. The PVC modulus has significant variability for commercial pipe, and this value is near the bottom of the range. The air sound speed is reduced by less than $0.1 \%$ by pipe wall compliance.

2.2. Initial Studies. Two initial permutations of the facility geometry were conducted at a constant main line flow velocity of $38 \mathrm{~m} / \mathrm{s}$ [12], corresponding to a flow Mach number of 0.111 . The position of the branch line, section 5 , was varied by changing the length of pipes 1 and 6 . They were changed such that the total length from the front edge of pipe 1 to the back edge of coupling 10 (Length B) remained constant at $297 \mathrm{~cm}$. Ten experiments were done such that the distance from the upstream boundary to the centre of the branch line was varied from $199 \mathrm{~cm}$ to $222 \mathrm{~cm}$ in $2.54 \mathrm{~cm}$ increments. The branch line was $16.0 \mathrm{~cm}$ long corresponding to $1 / 4$ wavelength frequency near $536 \mathrm{~Hz}$. A Hanning window is applied to each packet and a power spectral density (PSD) performed using Matlab. In initial tests, the peaks were examined manually. This was followed by capture and superposition of 20 PSDs from 2second windows sampled at $100 \mathrm{KSamples/second,} \mathrm{to} \mathrm{create}$ a plot like that shown in Figure 4.

In some situations the waveform produced by the branch line had a great deal of amplitude variation. Six of the ten configurations showed a single frequency peak near the expected value, like that shown in Figure 4. The other four had frequency content wandering over time, with 20 traces taking the form of Figure 5. Units are not reported on the $y$ axes of Figures 4 and 5 because the units were not available in [12]. However, Figures 4 and 5 remain useful in communicating the path of discovery and help explain the development of the signal handling methods used in this paper. 


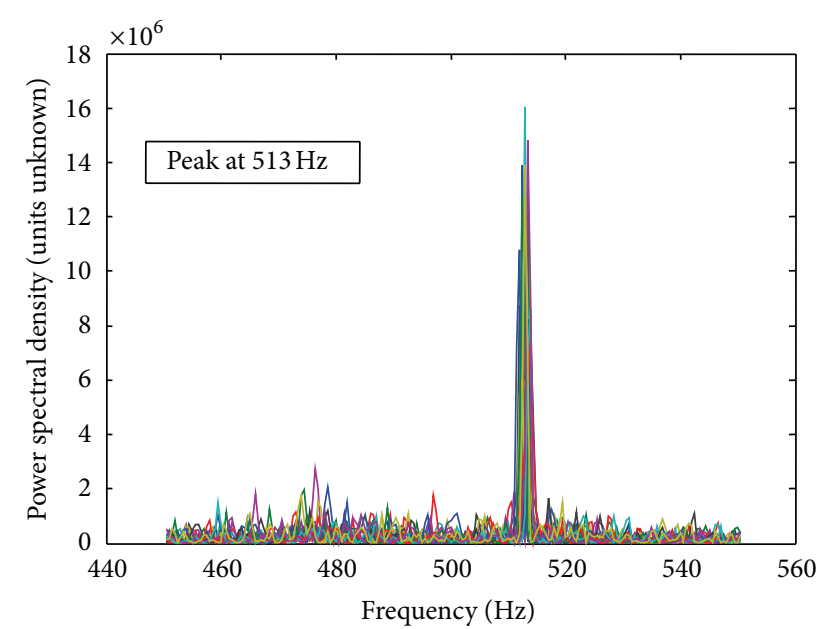

FIGURE 4: Twenty PSDs superimposed, early tests at constant $297 \mathrm{~cm}$ length. Taken from [12], PSD units unknown.

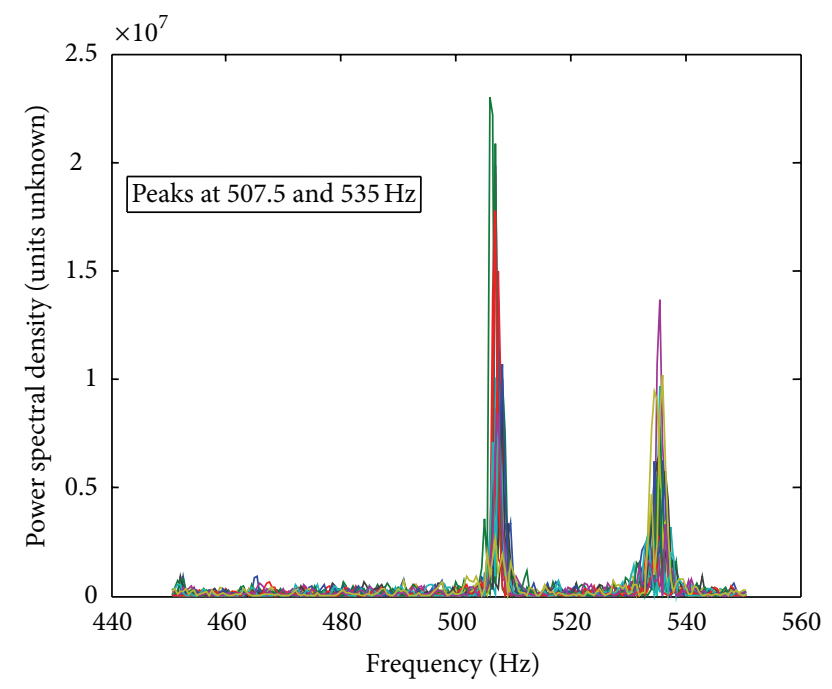

FIGURE 5: Twenty PSDs superimposed, early tests at constant $297 \mathrm{~cm}$ Length. Taken from [12] PSD units unknown.

Three individual PSDs derived from two-second time histories taken from one of the configurations with frequency variation are shown in Figure 6 to further illustrate how frequency content is varying in time for these tests.

Another set of experiments varied the position of the branch relative to the upstream boundary by changing the length of pipe 1 (Length A). However, the position of the branch relative to the downstream boundary remained constant, causing a change in the total length of the pipe (Length B). Ten pipe configurations were explored. In each successive configuration, pipe 1 was lengthened by $2.54 \mathrm{~cm}$ (1 inch), causing the distance from the upstream boundary to vary from 199 to $222 \mathrm{~cm}$. The distance from the pipe to the downstream boundary was held constant at $58.4 \mathrm{~cm}$. Twenty data packets of two seconds each were obtained for each piping configuration. The frequency content was measured and a typical 20 spectrum overlay for one main
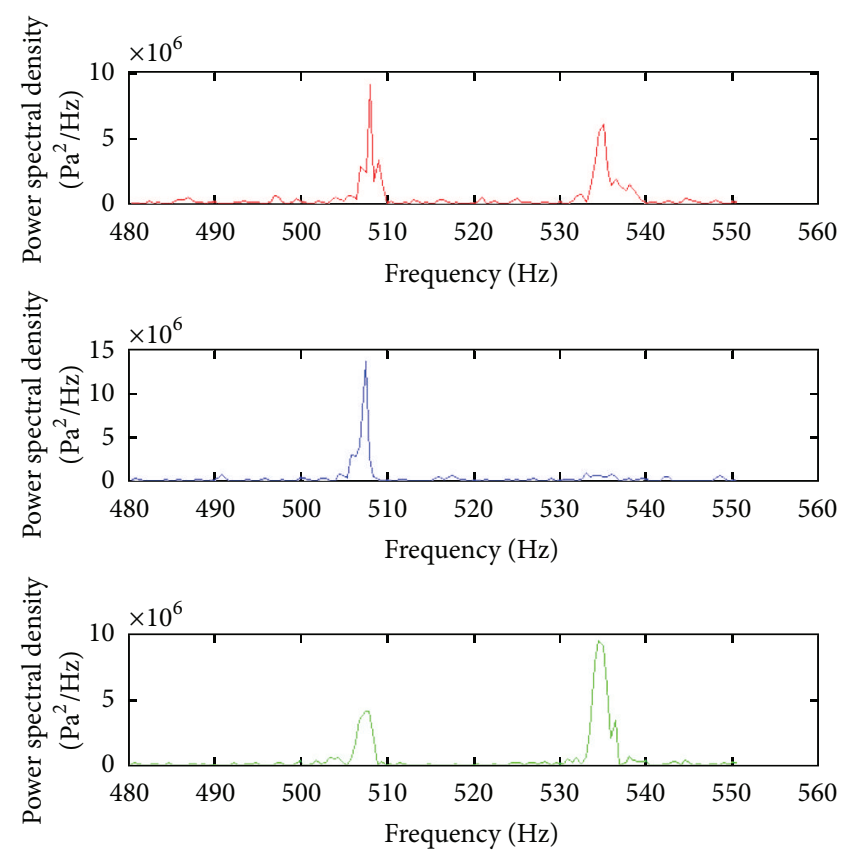

FIgURE 6: Three PSD from example variable frequency case.

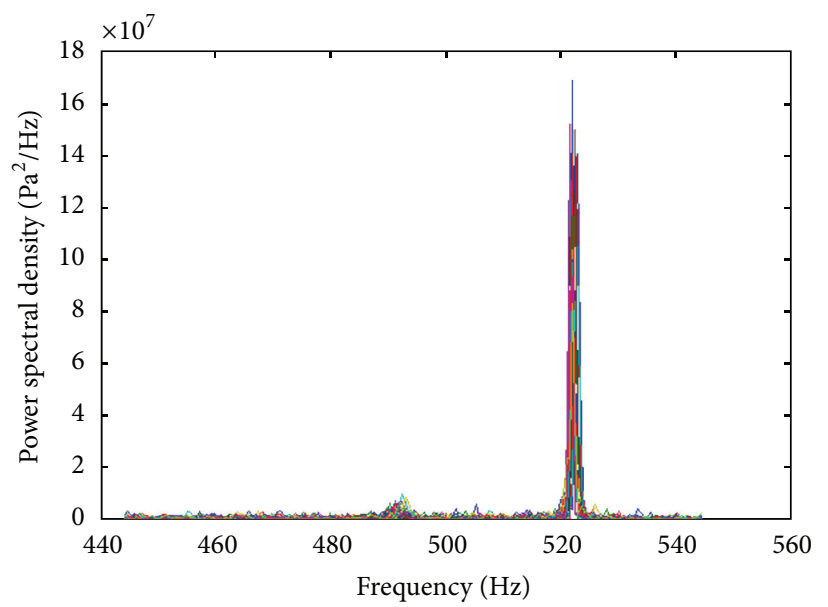

FIgURE 7: Typical PSD for Tests during Main Line Length Variation.

line length is shown in Figure 7. While the frequency of the peak was reasonably constant for each test, the value of the peak frequency varied with main line length, following the trend plotted in Figure 8. The peak frequency in the branch was the same as that measured in the main line for the cases recorded in Figure 8. Figure 8 suggests coupling between the main line resonance and side branch resonance, with the jump in frequency corresponding to a transition between $n$ and $n+1$ integer half wavelengths in the main line.

Initial velocity measurements used a Pitot tube, but the variability in acoustic amplitude data motivated several investigations of possible sources of variability, and redundant velocity measurement was implemented using heated film probes. The blower RPM was also measured early in the campaign for some tests to confirm steady blower operation. 


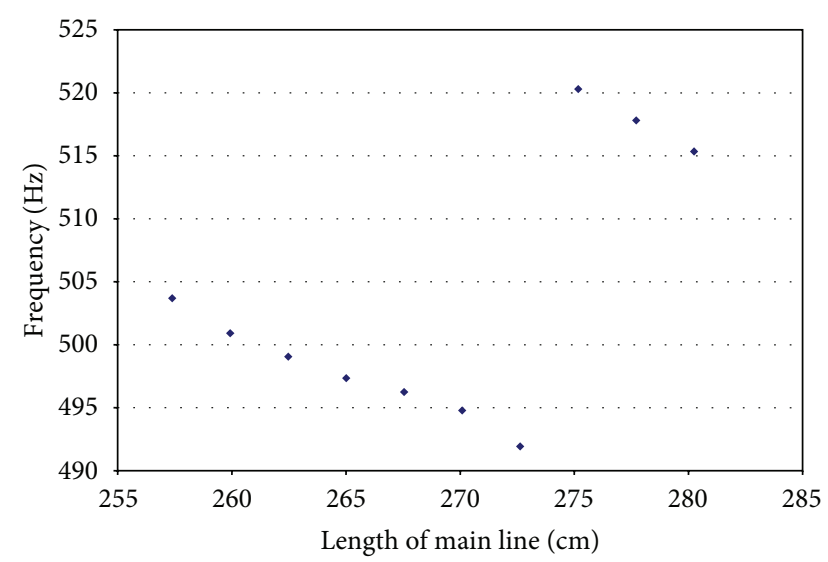

Figure 8: Peak frequency as a function of main line length.

Main line velocities were steady within the time response of the flow instruments, which was near 0.5 seconds for the film probes, and 0.02 seconds for the Pitot tube. The air bypass setting was fixed for all tests. The air temperature variations during the test campaign contributed to $1.5 \%$ variation in the sound speed. Bulk velocity measurement accuracy was within $3 \%$, with resolution within $0.5 \%$. The early studies exposed complex non-stationary acoustic behavior under confirmed steady flow conditions and led to the refinements in acoustic data handling techniques that follow.

2.3. Current Studies and Data Handling. The time variation in amplitude of peak frequencies observed in early tests was qualitatively consistent with observations in some of the acoustic data from nuclear power plants, and some method to characterize the variation in peak amplitude in the power plant data and in the air test data was needed. Many techniques exist to characterize non-stationary signals, but it was desired to choose a method that remained grounded in the frequency domain so that a connection to loading of structural components could be made using well established modal analysis methods.

The data interrogation method developed for this purpose uses a moving window technique. The method introduces a short time window that is moved across the pressure time history, and a PSD is developed for the data in each short window. The peak PSD amplitude and frequency is identified for each window, and the peak amplitude and frequency values from all the moving window positions are stored in arrays. The peak amplitude array is presented as a Probability Density Function (PDF) in this paper, but other representations are useful, such as correlation of amplitude with frequency. An average PSD is evaluated by adding PSD outcomes for all windows, and dividing by the total number of window evaluations. The peak amplitude of the average PSD and the PDF of the PSD peak amplitudes from the moving time windows are evaluated for each experiment.

Some example data are presented, where the total time history is 60 seconds, the moving window width is one second, and the data capture rate is 5,000 samples per second. The moving window is moved in one half second increments. The peak amplitude data from the PSD created by averaging

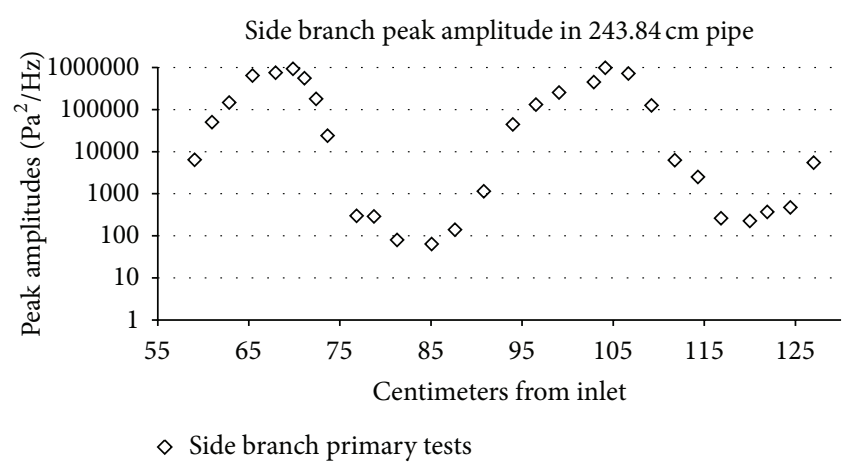

FIGURE 9: Average PSD amplitude in branch versus position along the main line.

119 PSDs from each 60-second time history is shown in Figure 9 as a function of branch position along the main line. A probability density function (PDF) is created from the array of moving window peak PSD amplitude values. The PDF and the average of moving window PSDs are provided for three specific measurement locations in Figures 10, 11, and 12 to show the amplitude variability and time average amplitude for those branch locations.

A microphone was deployed through the main line to measure the time average sound field. Figures 13, 14, and 15 show the time averaged PSD peak amplitude spatial distribution in the main line for three different branch locations. Figures 13 shows the PSD peak amplitude along the main line for a side branch position where the side branch resonance was near maximum. Figure 14 offers the PSD peak amplitude along the main line for a side branch position where the side branch resonance was of intermediate amplitude. Figure 14 offers the PSD peak amplitude along the main line for a side branch position where the side branch resonance was relatively weak. The spatial average PSD level in the main line trends with the amplitude of the side branch resonance. Also, no stationary standing wave pattern persisted in the main line for these cases. These PSD peak amplitudes occur for frequency corresponding to the branch resonance.

2.4. Data Handling Adequacy. The waveform produced by the branch line can have a great deal of variance in amplitude, and the length of the time history required to characterize the system behavior must be established. Figure 16 shows the average PSD for the $61 \mathrm{~cm}$ position. Figure 17 shows the variability in the running average peak amplitude value. This example shows that the average value has become reasonably stable after 60 seconds, or 119 window evaluations.

While average amplitude values are often adequate in characterizing acoustic system behavior, peak values and distributions of amplitude values are important if the pressure amplitudes are contributing to a component load history. Figure 17 indicates that a 60 -second time history is adequate to characterize the average value for this frequency and this time history. However, a more rigorous test may be appropriate to establish if the amplitude PDF is adequately characterized by the time history and to assure a near 

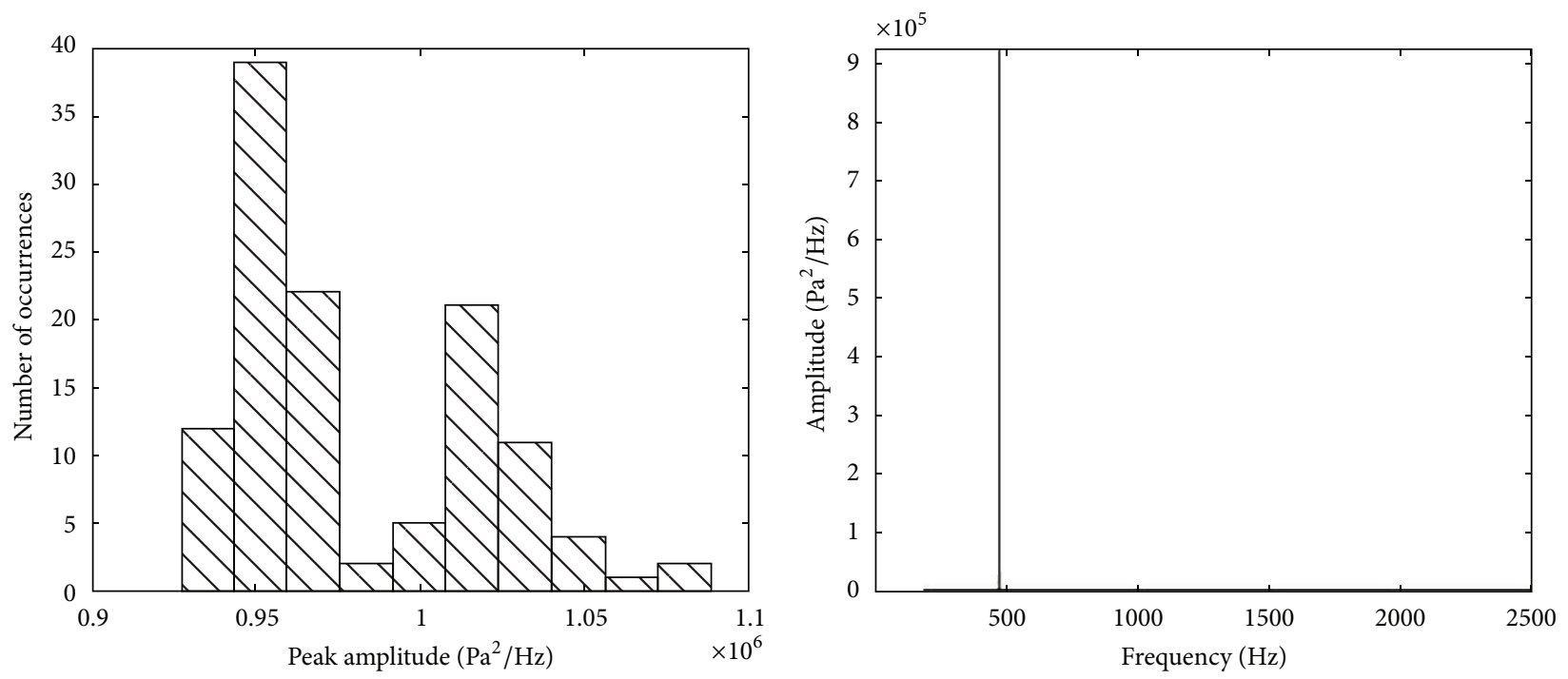

FIgUre 10: Amplitude PDF and average PSD $69.9 \mathrm{~cm}$ from inlet.
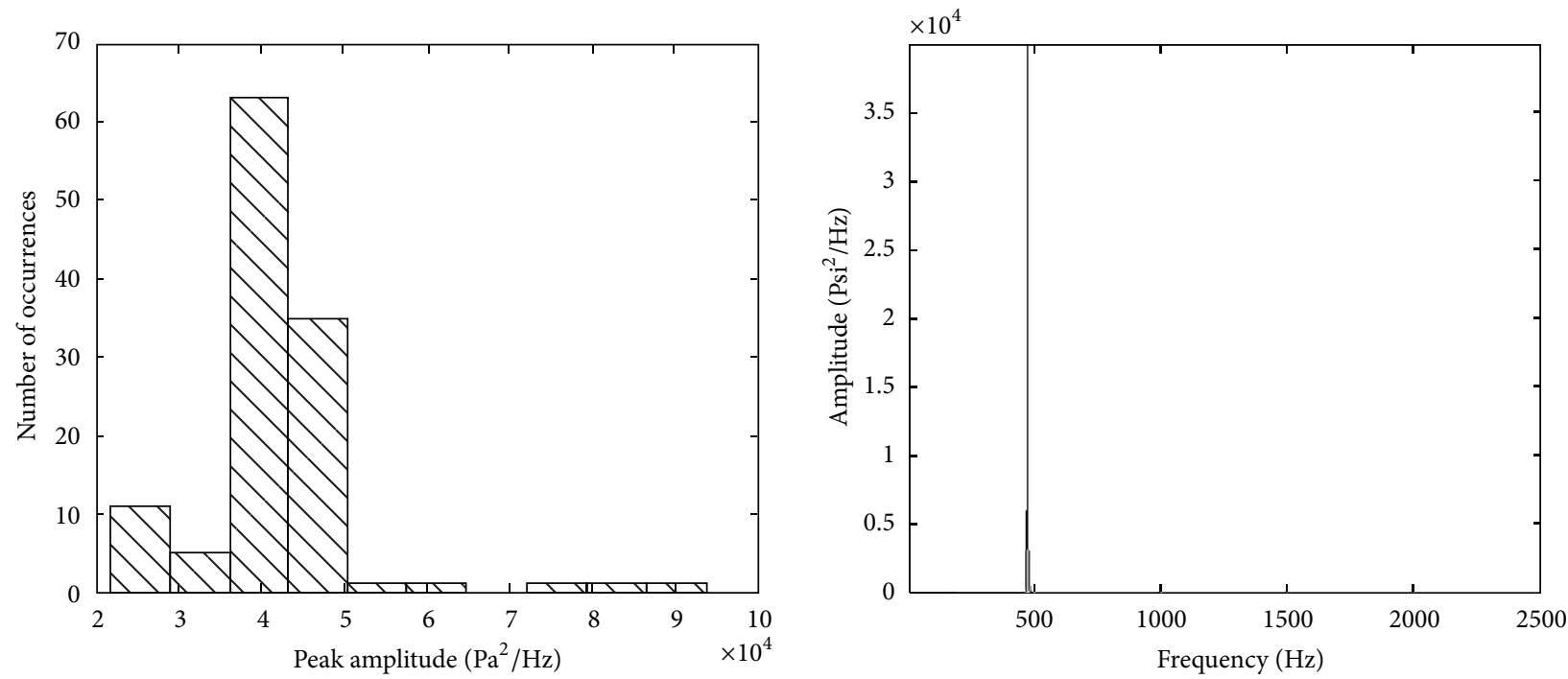

FIGURE 11: Amplitude PDF and average PSD $74.3 \mathrm{~cm}$ from inlet.

representative peak amplitude has been catalogued in the time history.

A two-second wide moving window, with one second overlap, and a $1 / 2$ second window with $1 / 4$ second overlap were examined before selecting the one second moving window width with $1 / 2$ second overlap used to characterize these data. The signal variability, as expressed in the PDF of the PSD amplitude peaks, did not increase as the time window size was decreased below one second. These data were collected with a fixed sample rate of 5,000 samples per second; so, the PSD frequency resolution is decreasing as the window size is reduced, and this may reduce peak values. The Hanning window used to condition the moving window attenuates the leading and trailing parts of the time history in the window, causing reported dynamic pressure amplitudes to be reduced from the actual values. Alternate methods could be developed that better preserve the pressure amplitude accuracy. The relatively smooth variation in resonance amplitude in time allows the shorter time histories, still containing several hundred resonant cycles, to be well represented using Fourier decomposition. This allows the load to be represented in the frequency domain, which is convenient to apply to structures in codes employing modal analysis.

The resonant pressure amplitudes measured in this facility vary greatly with branch position and vary significantly in time. Previous studies of side branch resonance in the literature suppressed the acoustic waves in the main line, and the side branch resonance was reported to be stationary over a range of Strouhal numbers. This makes a direct comparison with data from those studies impossible. However, one previous study does have facility attributes similar to those of the facility used here [13]. Those researchers varied flow velocity 

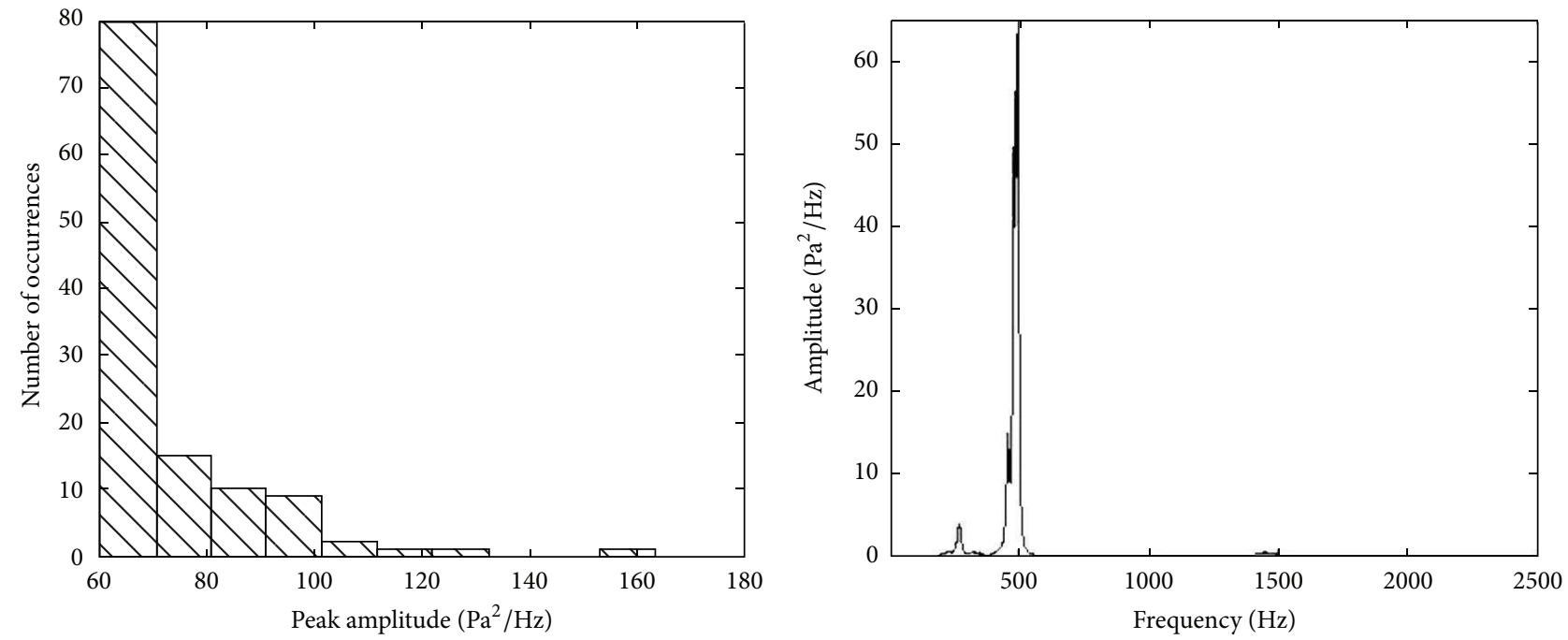

FIGURE 12: Amplitude PDF and average PSD $85.1 \mathrm{~cm}$ from inlet.

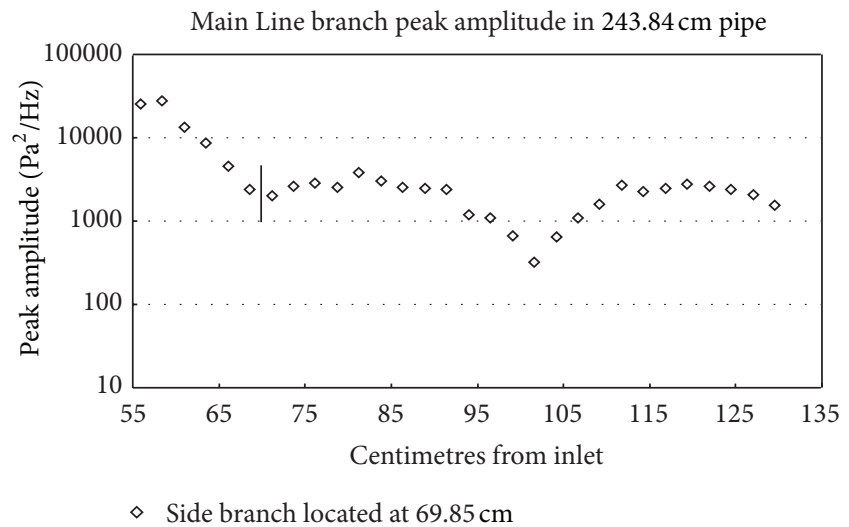

FIGURE 13: Main line waveform $69.9 \mathrm{~cm}$ from inlet.

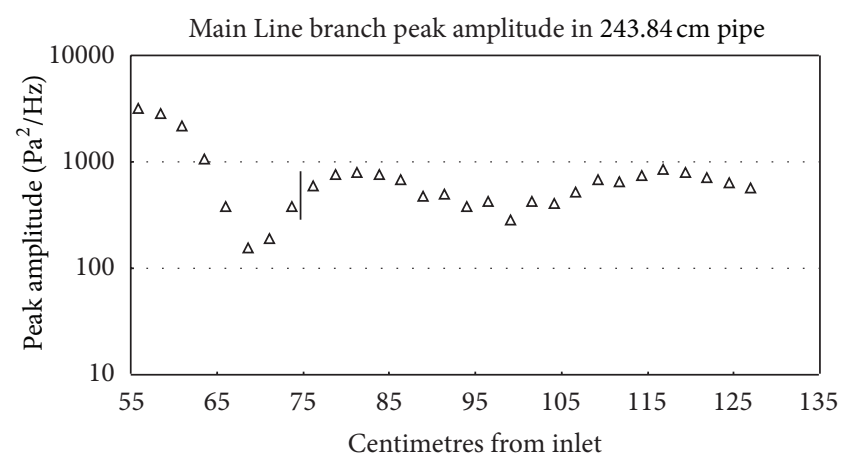

$\Delta$ Side branch located at $74.3 \mathrm{~cm}$

FIGURE 14: Main line waveform $74.3 \mathrm{~cm}$ from inlet.

such that the Strouhal number ranged from 0.3 to near 1.5 and then plotted the amplitude variation in the branch resonance pressure as a function of Strouhal Number. This approach follows the pattern of data collection typical of the

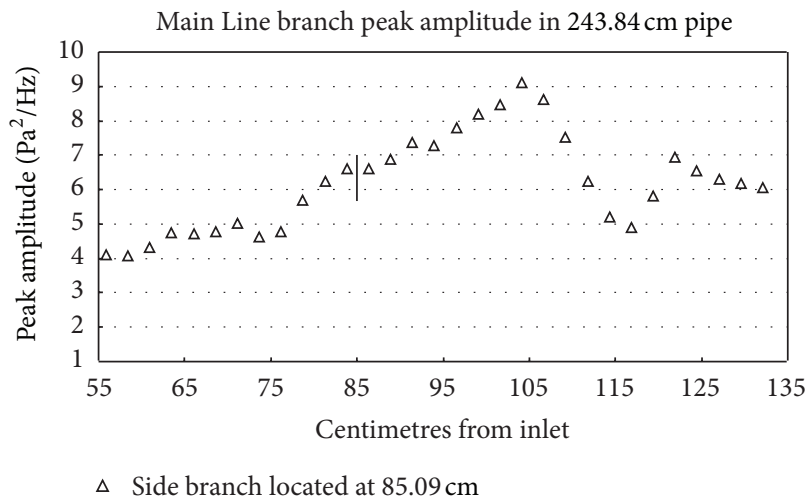

FIgURE 15: Main line waveform $85.1 \mathrm{~cm}$ from inlet.

literature, and a peak average sound pressure of 150 Decibels is reported near Strouhal equal 0.45 [13]. Peak average PSD values reported in Figure 9 range between $100,000 \mathrm{~Pa}^{2} / \mathrm{Hz}$ (143 Decibels) and 1,000,000 $\mathrm{Pa}^{2} / \mathrm{Hz}$ (154 Decibels).

\section{Conclusions}

Acoustic data are provided that characterize performance of a blocked side branch on a main line with flow. The branch diameter, $d=1.9 \mathrm{~cm}$, is positioned on a main line of diameter, $D=7.6 \mathrm{~cm}$, giving $d / D$ equal 0.25 . The vortex shedding at the branch is the energy source for acoustic waves in the branch and in the main line. Acoustic radiation from the entrance and exit of the main line is the primary acoustic energy sink for the system. The main line entrance and exit are compliant acoustic boundaries allowing accumulation of acoustic energy in the main line. The data show that the branch placement along the main line is very important to the amplitude of the branch resonance, and branch placement is important to the amplitude of the acoustic field in the main line. Some coupling of the branch and main line acoustic 


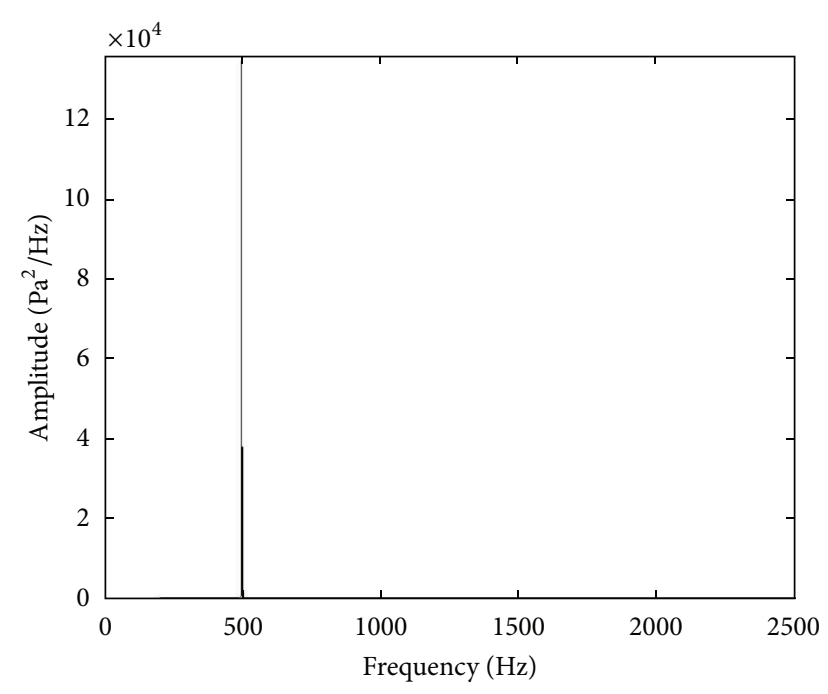

FIGURE 16: Average PSD for $61 \mathrm{~cm}$ position, one-minute history.

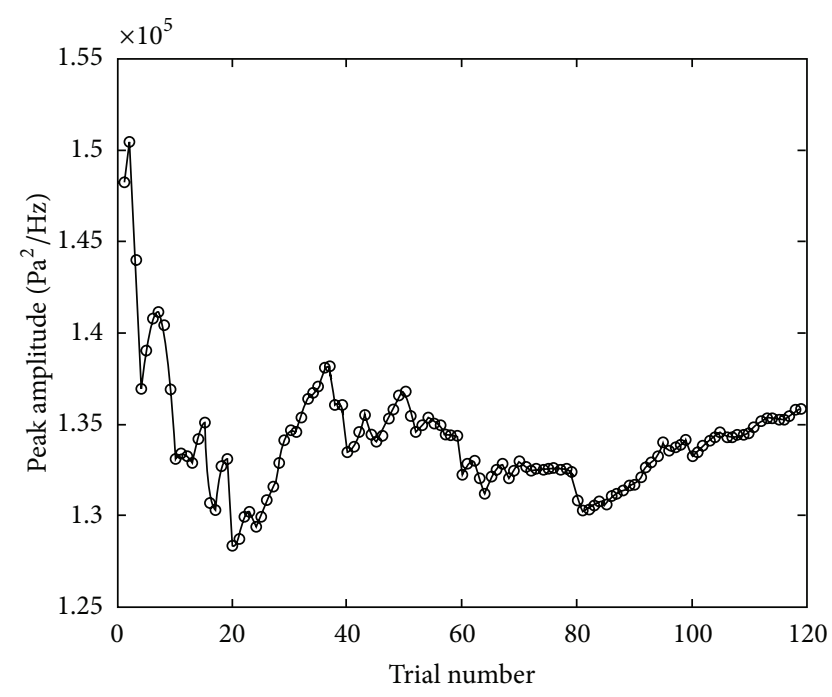

FIGURE 17: Running average of peak amplitude values.

performance is expected from acoustic circuit theory, but the extent of this influence on branch resonance has not previously been reported. The ratio of side branch acoustic pressure over flow dynamic pressure (i.e., the normalized amplitude ratio) achieves a value of 1.2 when the branch position is near $70 \mathrm{~cm}$ or $105 \mathrm{~cm}$ downstream of the inlet. The literature indicates normalized amplitude ratio at resonance near 0.2 for a single side branch with $d / D$ equal 0.25 when standing waves in the main line are suppressed [2].

Some locations of the branch along the main line are favorable to side branch resonance and create amplitudes in excess of those achieved when the main line standing waves are suppressed. This information is important to fluid transport system design since most fluid transport lines are not fitted with devices to suppress the main line acoustic waves. Other locations of the side branch along the main line greatly reduce the side branch resonance response. The main line acoustic wave response is also reduced in those cases, and a system with altogether greatly reduced acoustic energy is produced for otherwise identical branch geometry and flow conditions. This also is very useful information for fluid transport system design.

The main line and branch pressure signals are not stationary for most of the configurations that were tested. Most previous studies of side branch resonance in the literature deliberately suppress acoustic waves in the main line. This is likely why non-stationary signal attributes were not reported in those studies. The non-stationary attributes of the dynamic pressure signals encountered here made initial system characterization challenging. The approach to signal characterization eventually adopted and presented here collects amplitude peaks from a moving window spectrum evaluation in the form of a PDF. This method allows repeatable characterization of the non-stationary dynamic pressures this system produces. The non-stationary behavior of a side branch on a main fluid transport line has not been previously reported. However, there is one previous experiment in the literature with attributes similar to those of the facility used here that reported significant variability in branch resonance amplitude, as expressed by scatter in measured values, for one of the tested configurations [13].

While these signals are not stationary, the branch resonance amplitude is varying relatively smoothly and slowly in time, with a sample containing tens of cycles delivering consistent peak to peak amplitude. Many dynamic response and endurance evaluations for structural components are done in the frequency domain. Many structures are lightly damped, with damping of order $1 \%$. Fifty load cycles are required to integrate a structure mode to near full resonance amplitude when the structural damping is $1 \%$. Following the signal interrogation method presented herein, a window width of one second used for a one-minute time history could expose a resonance at $50 \mathrm{~Hz}$ existing for 50 to 100 load cycles. This peak resonance at $50 \mathrm{~Hz}$ may occur for only a few seconds in the one-minute history, while the majority of the time the amplitude could be significantly less. The peak amplitude values in the PDF from the moving window spectra allow connection of the non-stationary load attributes to a structural simulation using well-established and efficient modal analysis methods. A modal simulation can be performed using each load frequency and amplitude from the measured distribution to represent the dynamic stress in the structure. This approach also identifies segments of the longer time history that cause the most challenging structural loading. Methods like rain flow counting should be used if more rapid or spurious variation in the dynamic signal amplitude must be accommodated.

\section{References}

[1] D. Rockwell and E. Naudascher, "Self-sustained oscillations of impinging free shear layers," Annual Review of Fluid Mechanics, vol. 11, pp. 67-94, 1979.

[2] S. Ziada and S. Shine, "Strouhal numbers of flow-excited acoustic resonance of closed side branches," Journal of Fluids and Structures, vol. 13, no. 1, pp. 127-142, 1999. 
[3] P. N. Lamoureux and D. S. Weaver, "The effects of turbulence and damping on pipeline acoustic resonance," Proceedings of the Institution of Mechanical Engineers, vol. 416, pp. 303-312, 1991.

[4] S. Ziada, "Feedback control of globally unstable flows: impinging shear flows," Journal of Fluids and Structures, vol. 9, no. 8, pp. 907-923, 1995.

[5] S. Ziada and E. T. Buhlmann, "Multiple side-branches as tone generators," in Proceedings of International Mechanical Engineering Conference, paper 0416/009, pp. 435-444, 1991.

[6] D. S. Weaver and G. O. McLeod, Entrance Port Rounding Effects on Acoustic Resonance in Safety Relief Valves, vol. 389 of FlowInduced Vibration, PVP-ASME, 1999.

[7] J. T. Coffman and M. D. Bernstein, Failure of Safety Valves due to Flow Induced Vibration, Oklahoma Gas \& Electric Co. and Foster Wheeler Energy Corp., 1979.

[8] P. C. Kriesels, M. C. A. M. Peters, A. Hirschberg et al., "High amplitude vortex-induced pulsations in a gas transport system," Journal of Sound and Vibration, vol. 184, no. 2, pp. 343-368, 1995.

[9] J. C. Bruggeman, Flow induced pulsations in pipe systems [Ph.D. Dissertation], Technical University Eindhoven, 1987.

[10] M. C. A. M. Peters, E. van Bokhorst, and C. H. L. Limpens, "Impact of Flow Pulsations on Vortex Flow Meters," in Proceedings of the Flomeko, Sweden, 1998.

[11] A. E. Ruggles, “Technology Assessment of Boiling Water Reactor (BWR) steam supply acoustic performance and wet steam sound speed," Nuclear Technology, vol. 164, no. 3, pp. 309-319, 2008.

[12] P. McKinnis, B. Miller, and A. Ruggles, "Interaction between main line standing waves and side branch resonance frequencies," in 4th International Conference on Fluid Structure Interaction (Incorporating the Free and Moving Boundary Problems Seminar), pp. 337-349, gbr, May 2007.

[13] D. D. Ericson, W. W. Durgen, C. F. Maguire III, and M. J. Moeller, "Shear layer coupling with side branch resonators," in Proceedings of the ASME WAM, pp. 43-45, Anaheim, Calif, USA, 1986. 

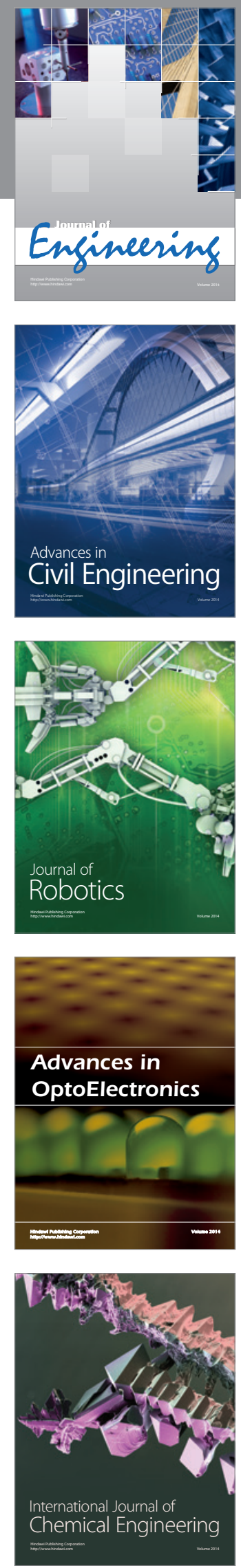

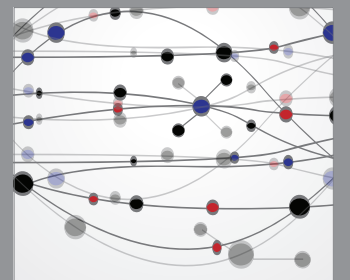

The Scientific World Journal
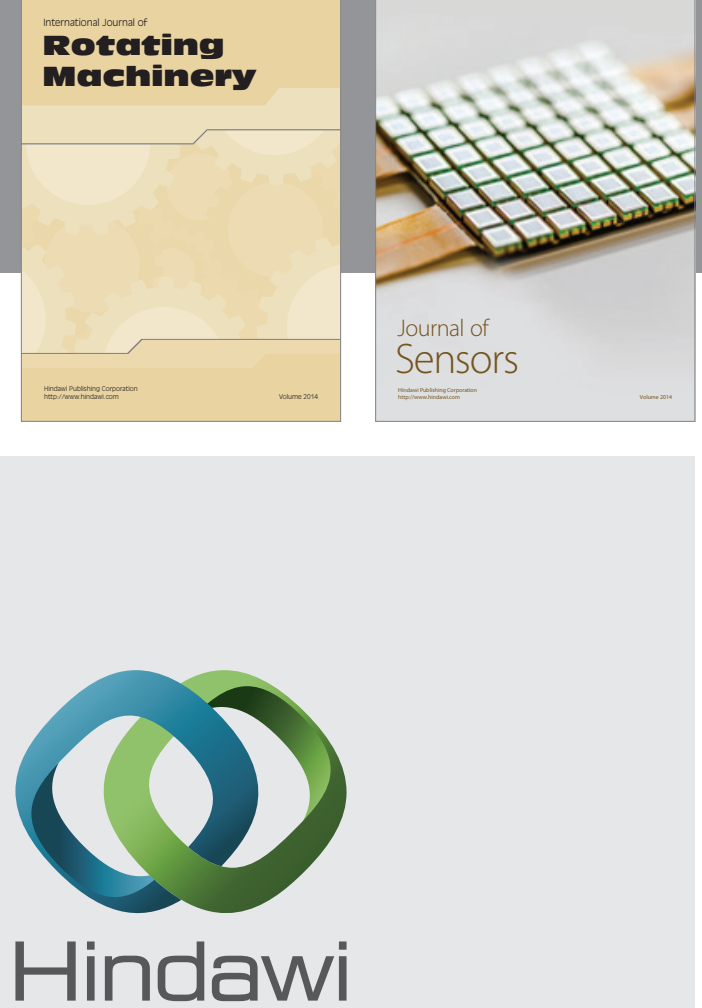

Submit your manuscripts at http://www.hindawi.com
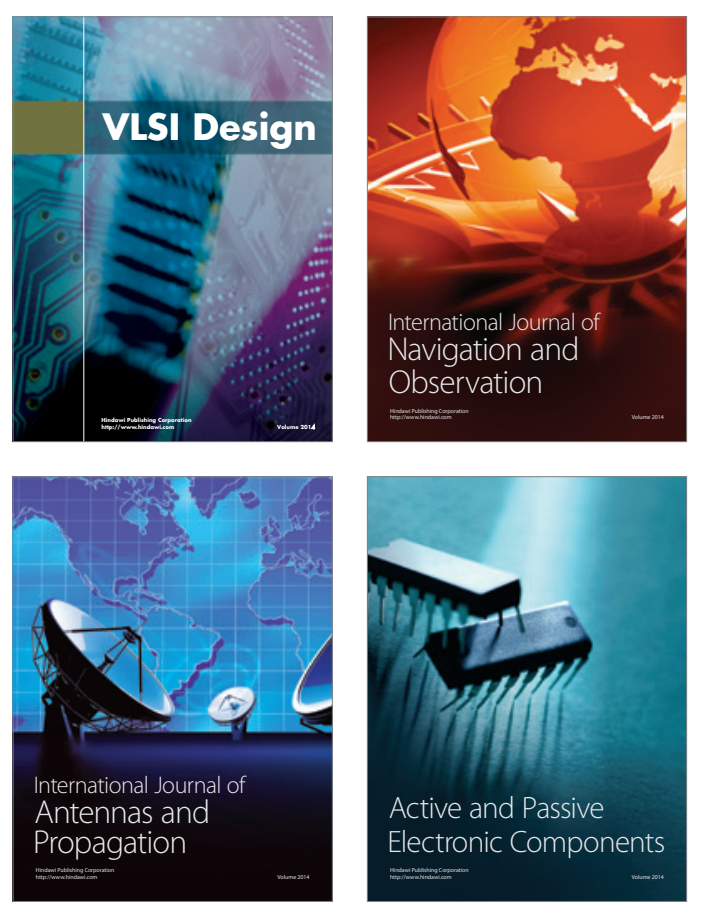
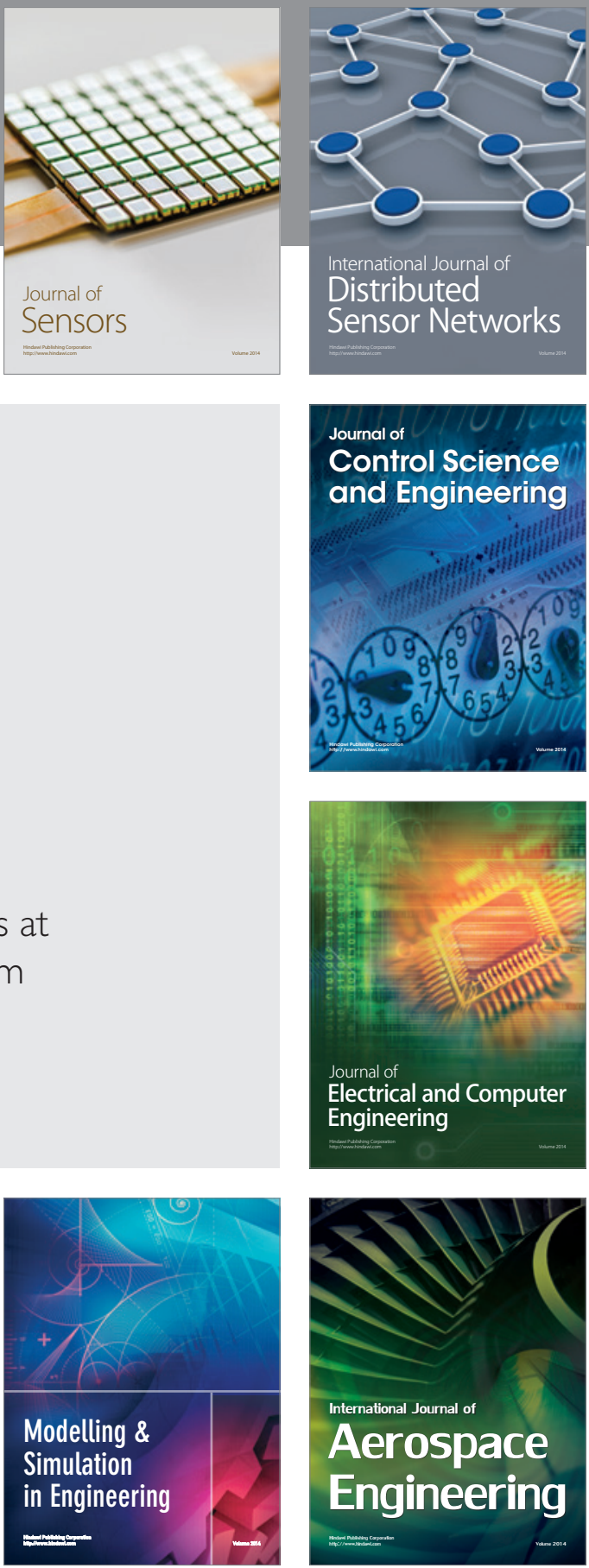

Journal of

Control Science

and Engineering
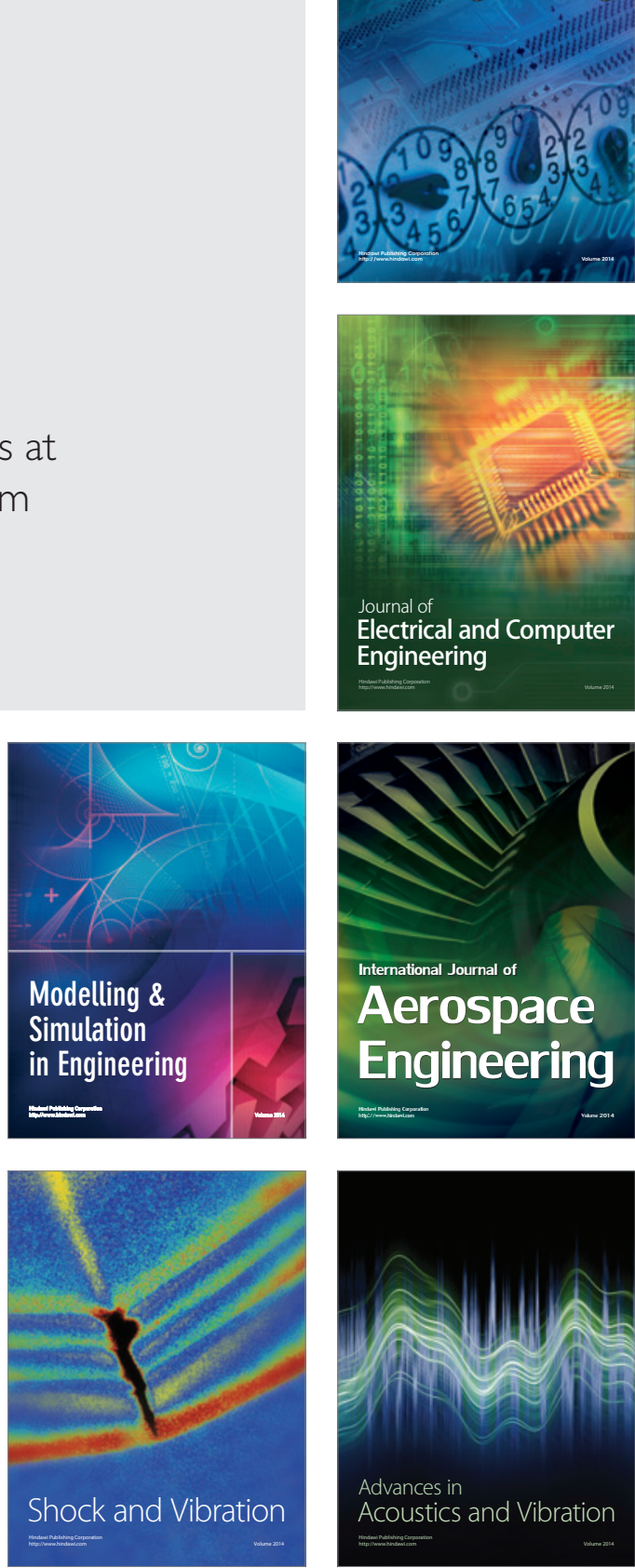